TAIWANESE JOURNAL OF MATHEMATICS

Vol. 12, No. 4, pp. 901-916, July 2008

This paper is available online at http://www.tjm.nsysu.edu.tw/

\title{
MORE ABOUT THE 52 FOUR-DIMENSIONAL PARALLELOTOPES
}

\author{
M. Deza and V. P. Grishukhin
}

Dedicated to Professor Ko-Wei Lih on the occasion of his 60th birthday.

\begin{abstract}
There are several works [6] (and [13]), [8], [2] and [14] enumerating four-dimensional parallelotopes. Engel [9] was the first who distinguished 17 zonotopal parallelotopes among them. Each zonotopal parallelotope is the Minkowski sum of segments whose generating vectors form a unimodular system. We show that there are exactly 17 four-dimensional unimodular systems. Hence there are 17 four-dimensional zonotopal parallelotopes. We prove that other 35 four-dimensional parallelotopes are: the regular 24-cell $\{3,4,3\}$ and 34 sums of the 24-cell with non-zero zonotopal parallelotopes. We give a detailed description of the construction of these 35 parallelotopes.
\end{abstract}

\section{INTRODUCTION}

A parallelotope is a convex polytope which fills the space facet to facet by its translation copies without intersecting by inner points. Such a filling by parallelotopes is a tiling. The centers of the tiles form a lattice. A parallelotope of dimension $n$ is called primitive if exactly $n+1$ adjacent parallelotopes meet in each vertex of its lattice tiling. Voronoi defines an L-type of a parallelotope, which is (in modern terms) the isomorphism class of the face order lattice of the parallelotope. A special kind of a parallelotope is the Voronoi polytope of a lattice. The Voronoi polytope at a lattice point $v$ is the set of points which are at least as close to $v$ as to any other lattice point. Voronoi conjectured that every parallelotope of a class of parallelotopes of the same L-type is affinely equivalent to a Voronoi polytope (of course, of the same L-type), and he proved this conjecture for primitive parallelotopes.

In four-dimensional space, Voronoi [17] determined all the 3 types of primitive parallelotopes. Using projection along a zone of parallel edges, Delaunay [6] found

Received August 23, 2007, Accepted November 12, 2007.

Communicated by Gerard J. Chang.

2000 Mathematics Subject Classification: 52C22; 51M20.

Key words and phrases: Parallelotopes, Voronoi polytope, Minkowski sum, root system $D_{n}$, Unimodular systems. 
51 types of four-dimensional parallelotopes. The missed 52th type was discovered by Stogrin [13].

Engel verified by computer the result of Delaunay corrected by Stogrin. In Table 9.4 of [8] (see also Table 1 of [9]) he gives an informative and useful list of parallelotopes of the 52 types. Besides, in Fig.9.7 of [8] he gives a partial order between the 52 parallelotopes. This order consists of two disjoint components. Recall, that a zonotope is the Minkowski sum of segments. Results of our paper imply, that these two partial orders correspond to partial orders between zonotopes: the first one between zonotopes which are themselves parallelotopes and the second one between zonotopes the Minkowski sum of which with the 24-cell gives a parallelotope.

Conway in the chapter "Afterthoughts: Feeling the form of a Four-dimensional lattice" of [2] proposes conorms for to characterize shapes (i.e. types) of parallelotopes. (In fact, Conway enumerates shapes of four-dimensional Voronoi polytopes.) The 17 types of parallelotopes which are zonotopes are parameterized by 16 subgraphs of the complete graph $K_{5}$ and by the complete bipartite graph $K_{3,3}$. The remaining 35 types of parallelotopes are characterized by shapes of positions of minimal conorms in $4 \times 4$ matrices of all conorms. Vallentin [14]repeated the above Conway's computations. He computes in details the 35 nonequivalent conorms.

In [12] Ryshkov asserts that a four-dimensionl parallelotope is either the Minkowski sum of segments or the Minkowski sum of the 24-cell with a set of segments. The segments of the set are parallel to the edges of the 24-cell. But no proof of these assertions was published. Using Theorems 1 and 2 (see Section 2 below), we give here a detailed proof of these assertions.

Recall that a set of vectors $U$ is a unimodular system if every vector $u \in U$ has an integer representation in any basic subset $B \subseteq U$ (details see, for example, [5]). McMullen [11] proved that an $n$-dimensional zonotope is a parallelotope if and only if the directing vectors of its segments span an $n$-dimensional unimodular system.

It is well known (see, for example, [5]) that there are two maximal fourdimensional unimodular systems. These are the graphic system of 10 vectors representing the regular matroid of the complete graph $K_{5}$ and the cographic system of 9 vectors representing the cographic matroid of the complete bipartite graph $K_{3,3}$. All matroid definitions given here and below can be found in any book on Matroid Theory, for example in [1].

Each subsystem of the maximal cographic unimodular system $K_{3,3}^{*}$ is graphic and represents a subgraph of $K_{5}$. Hence each four-dimensional zonotopal parallelotope is generated either by one of 16 graphic unimodular subsystems of $K_{5}$ or by the cographic system $K_{3,3}^{*}$. Note that the graph $K_{4}$ in the table on page 87 of [2] generates a three-dimensional unimodular system. But the rank 4 subgraph $C_{221}+1$ of $K_{5}$ is missed in the Conway's table. (An explanation of the denotation $C_{221}+\mathbf{1}$ 
is given below in Section 3.) Note that in [14], zonotopes are described by their facet vectors. The facet vectors of a zonotope $Z(M)$ related to a matroid $M$ form a representation of the dual matroid $M^{*}$. Hence, in contrast to us, Vallentin considers cographic matroids of corresponding graphs.

We show that the remaining 35 parallelotopes are Minkowski sums of the regular 24-cell $\{3,4,3\}$ with zonotopes related to unimodular systems and the 24-cell itself.

We use heavily two important theorems from [10] (Theorems 1 and 2 below). These theorems allow us to describe in details all possibilities to add segments to the 24-cell for to obtain a parallelotope. Note that the same unimodular system can give distinct sums with the 24-cell. We explain how this happens. In fact, we show that the 24-cell uniquely determines the 34 sums of it with zonotopal parallelotopes.

Until now, all enumerations asserted only that there exist 52 four-dimensional parallelotopes. In our paper, we explain why there are only 52 four-dimensional parallelotopes.

We proceed as follows. For the 24-cell, which is the Voronoi polytope $P_{V}\left(D_{4}\right)$ of the root lattice $D_{4}$, we find the set of all, up to multipliers, vectors $z$ such that the sum of $P_{V}\left(D_{4}\right)$ with a segment spanned by $z$ is a parallelotope. This set is finite. In this set we consider subsets which span unimodular systems. Amongst them we separate subsets $Q$ such that the sum of $P_{V}\left(D_{4}\right)$ with the zonotope generated by $Q$ is a parallelotope. This algorithm gives all the 34 sums of the 24-cell with zonotopal parallelotopes.

The case of four-dimensional parallelotopes is a very instructive example of constructions of a large class of $n$-dimensional parallelotopes.

\section{Parallelotopes of Non-zero Width}

Venkov introduced in [16] a notion of a polytope of non-zero width in direction of a $k$-dimensional subspace $X^{k}$ as a polytope whose intersection with any affine $k$-space parallel to $X^{k}$ is either $k$-dimensional or empty. He studied parallelotopes of non-zero width. The most interesting are parallelotopes of non-zero width in direction of a line (or in direction of a vector spanning this line).

It is not difficult to see that if a parallelotope $P$ has a non-zero width in direction of a line $l$, then the line $l$ is parallel to some edges of $P$. A set of mutually parallel edges of $P$ is called an edge zone of $P$. Following Delaunay [6], Engel called an edge zone closed if each two-dimensional face of $P$ has either two or none of edges of this zone. Otherwise, the zone is called open.

In [10], the following Proposition is proved.

Proposition 1. For a parallelotope $P$ and a vector $z$, the following assertions are equivalent:

(i) P has a closed edge zone parallel to z; 
(ii) $P$ has a non-zero width in direction of $z$;

(iii) $P$ is the Minkowski sum of a segment $S(z)$ of the line spanned by $z$ and a parallelotope $P^{\prime}$ of zero width in direction $z$, i.e., $P=P^{\prime}+S(z)$.

We say that a parallelotope $P$ is of (or has) zero width in direction $z$ if $P$ is not of non-zero width in this direction. The length of the segment $S(z)$ in item (iii) is equal to the length of the shortest edge of the closed zone parallel to $z$. It implies that this zone is open in the parallelotope $P^{\prime}$. Note that $S(z)=\lambda(z-z)$ for some real $\lambda$, where $z-z=\{x=\alpha z:-1 \leq \alpha \leq 1\}$ is the Minkowski sum of $z$ and $-z$.

If the parallelotope $P^{\prime}$ in the sum $P=P^{\prime}+S(z)$ is also of non-zero width in another direction $z_{1}$, then, by Proposition 1(iii), $P^{\prime}=P^{\prime \prime}+S\left(z_{1}\right)$. Of course, the Minkowski sum is associative and distributive. Hence, if a parallelotope $P$ has a non-zero width in several directions $z \in Q$, then $P=P_{0}+\sum_{z \in Q} S(z)$, where $P_{0}$ is a parallelotope, which has no direction of non-zero width. The sum $Z(Q)=\sum_{z \in Q} S(z)$ is a zonotope. If the original parallelotope $P$ is a zonotope, then $P_{0}$ is a point and $P=Z(Q)$.

We say that the set of vectors $Q$ spans $U$ if there are scalars $\beta_{z}, z \in Q$, such that $U=\left\{\beta_{z} z: z \in Q\right\}$. It is proved in [10] the following theorem.

Theorem 1. Let $Q(P)$ be the set of vectors along all directions of non-zero width of a parallelotope $P$. The set $Q(P)$ spans a unimodular system $U(P)$.

Since the zonotope $Z(Q)$ has a non-zero width along each $z \in Q$, Theorem 1 implies the result of McMullen [11] that the zonotope $Z(Q)$ is a parallelotope if and only if $Q$ spans a unimodular system.

The Proposition 1 shows that, sometimes, one can add a segment to a parallelotope $P_{0}$, in order to obtain another parallelotope. If the parallelotope $P_{0}$ has zero width in direction $z$, then the parallelotope $P_{0}+S(z)$ has another L-type than the original one. In [10] necessary and sufficient conditions are given, when the sum of a parallelotope with a segment is a parallelotope. In order to formulate these conditions, we introduce some new notions.

Venkov [15] proved that a polytope $P$ is a parallelotope if and only if $P$ itself and all its facets are centrally symmetric and the projection of $P$ along any $(n-2)$ dimensional face is either a parallelogram, or a centrally symmetric hexagon. The four or six facets, which are projected into edges of a parallelogram or of a hexagon form a 2-belt or a 3-belt, respectively.

A facet $F$ of a parallelotope $P$ is defined by a facet vector $p$, such that the facet $F$ lies in the affine hyperplane $\left\{x \in \mathbf{R}^{n}: p^{T} x=\frac{1}{2} p^{T} t\right\}$. Here $t$ is the lattice vector connecting the center of $P=P(0)$ with the center of the parallelotope $P(t)$ adjacent to $P$ by the facet $F$. Let $I$ be the set of indices of all pairs of opposite 
facets of $P$. Then we have:

$$
P(0)=\left\{x \in \mathbf{R}^{n}:-\frac{1}{2} p_{i}^{T} t_{i} \leq p_{i}^{T} x \leq \frac{1}{2} p_{i}^{T} t_{i}, i \in I\right\} .
$$

If $P=P(0)$ is a Voronoi polytope, then the facet vectors $p_{i}$ are parallel to the lattice vectors $t_{i}$, and we can set $p_{i}=t_{i}$. We use the same names 2- and 3-belts for the two and three facet vectors, defining facets of a 2- and a 3-belt, respectively.

It is proved in [10] the following important theorem.

Theorem 2. For a parallelotope $P$ and a vector $z$, the following assertions are equivalent:

(i) the Minkowski sum $P+S(z)$ is a parallelotope;

(ii) vector $z$ is orthogonal to at least one facet vector of each 3-belt of $P$.

\section{The Voronoi Polytope of the Lattice $D_{n}$}

We apply Theorem 2 to the Voronoi polytope $P_{V}\left(D_{n}\right)$ of the root lattice $D_{n}$. We find here all vectors $z$ such that $P_{V}\left(D_{n}\right)+S(z)$ is a parallelotope.

The facet (and lattice) vectors of the Voronoi polytope $P_{V}\left(D_{n}\right)$ are $n(n-1)$ roots of the root system $D_{n}$. We take the roots in the usual form $e_{i} \pm e_{j}, 1 \leq i<j \leq n$. Here $\left\{e_{i}: i \in N=\{1,2, \ldots, n\}\right\}$, is an orthonormal basis of $\mathbf{R}^{n}$. According to (1), we have:

$$
P_{V}\left(D_{n}\right)=\left\{x \in \mathbf{R}^{n}:-1 \leq x_{i} \pm x_{j} \leq 1,1 \leq i<j \leq n\right\} .
$$

For $n \geq 3$, the vertices of $P_{V}\left(D_{n}\right)$ are of the following two forms (cf. [3], p. 90):

$$
\pm v_{i}= \pm e_{i}, i \in N, \text { and } v(S)=\frac{1}{2}(e(S)-e(\bar{S})), S \subseteq N,
$$

where $\bar{S}=N-S$ and $e(T)=\sum_{i \in T} e_{i}$ for any $T \subseteq N$. The $2^{n}$ vertices of the set $\{v(S): S \subseteq N\}$ are vertices of a unit cube with its center in origin. The $2 n$ vertices $\pm v_{i}, i \in N$, form $2 n$ pyramids having the $2 n$ facets of the unit cube as bases. Hence, the vertex $v_{i}$ is adjacent to a vertex $v(S)$ only if $S \ni i$. Similarly, the vertex $-v_{i}=-e_{i}$ is adjacent to a vertex $v(S)$ only if $i \notin S$. The edge $v(S)-v_{i}=\frac{1}{2}(e(T)-e(\bar{T})), T=S-\{i\}$, connects these vertices. The vertex $v(S)$ is adjacent to a vertex $v\left(S^{\prime}\right)$ only if $n \geq 4$ and $v(S)-v\left(S^{\prime}\right)= \pm e_{i}$ for some $i \in N$.

So, edges of $P_{V}\left(D_{n}\right)$ are parallel to vectors of the following form

$$
e(S)-e(\bar{S}), S \subseteq N, \text { and } e_{i}, i \in N
$$


Up to sign, there are $2^{n-1}+n$ directions of edges, i.e., edge zones.

Each facet of $P_{V}\left(D_{n}\right)$ is an $(n-1)$-dimensional bipyramid with an $(n-2)$ dimensional cube as its base. Hence, each 2-face of $P_{V}\left(D_{n}\right)$ is a triangle. This implies that all edge zones of $P_{V}\left(D_{n}\right)$ are open.

Proposition 2. The following assertions are equivalent:

(i) $P_{V}\left(D_{n}\right)+S(z)$ is a parallelotope;

(ii) $z$ is parallel to an edge of $P_{V}\left(D_{n}\right)$.

Proof. (i) $\Leftrightarrow$ (ii). By Theorem 2, we have to show that a vector $z$ is orthogonal to at least one facet vector $p$ of each 3-belt of $P_{V}\left(D_{n}\right)$ if and only if it is parallel to an edge zone of $P_{V}\left(D_{n}\right)$.

The 3-belts of $P_{V}\left(D_{n}\right)$ are of the following two types:

$$
\text { (a) } e_{i}-e_{j}, e_{j}-e_{k}, e_{i}-e_{k} ;(b) e_{i}+e_{j}, e_{j}+e_{k}, e_{i}-e_{k} .
$$

We find all vectors $z=\sum_{i=1}^{n} z_{i} e_{i}$ such that $z^{T} p=0$ for at least one facet vector $p$ of each belt. The vector $z$ cannot have 3 mutually non-equal coordinates. In fact, if there are three such coordinates $z_{i}, z_{j}, z_{k}$, then $z$ is not orthogonal to any facet vector of the belt $\left(e_{i}-e_{j}, e_{j}-e_{k}, e_{i}-e_{k}\right)$ of type (a). Therefore, the vector $z$ should be of the form $z=z^{\prime}(S):=z_{1} e(S)+z_{2} e(\bar{S}), S \subseteq N$. Since at least one pair of each triplet of indices $\{i, j, k\}$ lies either in $S$, or in $\bar{S}$, the vector $z^{\prime}(S)$ is orthogonal to at least one vector of each belt of type (a).

For the vector $z^{\prime}(S)$ to be orthogonal to at least one vector of each belt of type (b), it should be either $z_{1}=z_{2}$, or $z_{1}=0$ and $|\bar{S}|=1$, or $z_{2}=0$ and $|S|=1$, or $z_{1}+z_{2}=0$. The last condition is necessary for the vector $z^{\prime}(S)$ with $z_{1} \neq z_{2}$ to be orthogonal to at least one vector of the belt of type (b), such that either $i \in S$, $k \in \bar{S}$, or $k \in S, i \in \bar{S}$.

So, we obtain that, up to a multiplier, the vector $z$ has one of the form

$$
z=z(S)=e(S)-e(\bar{S}), S \subseteq N, \text { or } z=e_{i}, i \in N .
$$

Comparing these vectors with vectors from (2) of edges of $P_{V}\left(D_{n}\right)$, we obtain that all vectors $z$ are directed along edges of $P_{V}\left(D_{n}\right)$.

\section{Four-dimensional Unimodular Systems and Zonotopal Parallelotopes}

Let $\Sigma_{n}$ be an $n$-dimensional simplex. It has $\frac{1}{2} n(n+1)$ edges. The $\frac{1}{2} n(n+1)$ vectors which are parallel to edges of $\Sigma_{n}$ and have the same length as corresponding edges, form a maximal unimodular system $A_{n}$. It represents the graphic matroid of the complete graph $K_{n+1}$ which is the one-dimensional skeleton of the simplex 
$\Sigma_{n}$. Recall that a set of vectors corresponding to edges of a graph $G$ represents a graphic (cyclic) matroid of the graph $G$ if the sum of vectors (taken in suitable direction) along any cycle of $G$ is zero vector. Changing in this definition cycle by cocycle (cut), we obtain a representation of the cographic matroid of $G$. (See any book on Matroid Theory, for example, [1].)

We identify the vectors of $A_{n}$ with the corresponding edges of $\Sigma_{n}$ and $K_{n+1}$. Take the $n$ vectors incident to a vertex $v \in \Sigma_{n}$ as a basis of $A_{n}$ and denote them $e_{i}, 1 \leq i \leq n$. Suppose that these vectors are directed from the vertex $v$. Then the other $\frac{1}{2} n(n-1)$ vectors of $A_{n}$ are $e_{i}-e_{j}, 1 \leq i<j \leq n$.

The Minkowski sum of all vectors of $A_{n}$ is an $n$-dimensional zonotope which is called permutohedron. It is the Voronoi polytope of the dual root lattice $A_{n}^{*}$ (see [3], p. 88). Besides, it is a primitive parallelotope. Voronoi called its L-type as the principal type.

Unimodular $n$-dimensional subsystems of $A_{n}$ are related to subgraphs of rank $n$ of $K_{n+1}$. Recall that rank of a graph is the number of its vertices minus the number of its components.

If a graph $G$ is planar, then there exists its dual planar graph $G^{+}$edges of which are in one-to-one correspondence with edges of $G$. The graphic matroid of $G$ is isomorphic to the cographic matroid of $G^{+}$. Both these matroids are represented by a common unimodular system.

A deletion of an element from the cographic matroid of a graph $G$ provides the contraction of the corresponding edge of $G$. It means that the end vertices of the contracted edge are identified and the obtained loop is deleted. For the graph $K_{3,3}$, the contraction of an edge gives a planar graph on 5 vertices. This graph is the subgraph of $K_{5}$ obtained by deletion from $K_{5}$ of two non-adjacent edges. It is denoted by $K_{5}-2 \times \mathbf{1}$. The dual $\left(K_{5}-2 \times \mathbf{1}\right)^{+}$is isomorphic to $K_{5}-2 \times \mathbf{1}$.

Here and below instead of the sum $1+1+\ldots+1$ of $k$ ones of [2] (denoting $k$ non-adjacent edges), we write $k \times 1$. We use here and below the following Conway's denotations: $C_{i j k \ldots}$ denotes the graph consisting of more than two chains each containing $i, j, k, \ldots$ edges and all chains connect the same two vertices. But note that $C_{k}$ is a cycle with $k$ edges, i.e. $C_{k}=C_{i j}$ with $i+j=k . G+k \times \mathbf{1}$ denotes a graph $G$ with $k$ pendant edges. For the matroid of the graph $G+k \times \mathbf{1}$, it is not important, whether the $k$ edges are connected to $G$ or not, or the $k$ edges form a tree or they are disconnected. It is important, that the subgraph induced by these $k$ edges contains no cycle.

In dimension 4, there are two maximal unimodular systems:

(1) $A_{4}$, representing the graphic matroid of the complete graph $K_{5}$, and

(2) the unimodular system, representing the cographic matroid $K_{3,3}^{*}$ of the complete bipartite graph $K_{3,3}$.

There are 16 subgraphs of rank 4 in $K_{5}$. They are drawn on p.87 of [2]. But 
the graph $K_{4}$ on this picture has rank 3. It should be changed by the subgraph $C_{221}+1$ missed in [2]. A corect picture of these graphs is given on p.55 of [14].

Since proper cographic submatroids of $K_{3,3}^{*}$ are isomorphic to graphic ones, in dimension 4 , there is only one cographic unimodular system $K_{3,3}^{*}$, which is not isomorphic to graphic one. Hence, besides the mentioned above 16 graphic fourdimensional unimodular systems, there is the 17th cographic four-dimensional unimodular system $K_{3,3}^{*}$. This implies, there are exactly 17 four-dimensional zonotopal parallelotopes. Amongst them only permutohedron is primitive.

Note that all edge zones of a zonotope are closed. All edges of an edge zone of a zonotope have the same length. A deletion of a vector from the unimodular system of a zonotope relates to contraction of the corresponding edge zone.

The correspondence of zonotopal paralelotopes from [6] with subgraphs of $K_{5}$ is given in Table 1. In this table, $N_{D}$ denotes the number given to a parallelotope in [6] (we call it Delaunay number), and $m$ is the number of segments in the Minkowski sum of the corresponding zonotope. According to [2], 2 and $\mathbf{3}$ denote the subgraphs of $K_{5}$, which are connected chains of two and three edges, respectively.

Table 1. Four-dimensional zonotopal parallelotopes $Z(G)$

\begin{tabular}{|c||c||c|c||c|c||c|c|c|c||}
\hline$N_{D}$ & 1 & 4 & 19 & 5 & 6 & 7 & 8 & 9 & 10 \\
\hline$m$ & 10 & 9 & 9 & 8 & 8 & 7 & 7 & 7 & 7 \\
\hline$G$ & $K_{5}$ & $K_{5}-\mathbf{1}$ & $K_{3,3}^{*}$ & $K_{5}-2 \times \mathbf{1}$ & $K_{5}-\mathbf{2}$ & $K_{5}-\mathbf{1}-\mathbf{2}$ & $K_{4}+\mathbf{1}$ & $C_{2221}$ & $K_{5}-\mathbf{3}$ \\
\hline
\end{tabular}

\begin{tabular}{|c||c|c|c|c||c|c|c||c||}
\hline$N_{D}$ & 11 & 12 & 13 & 16 & 14 & 15 & 17 & 18 \\
\hline$m$ & 6 & 6 & 6 & 6 & 5 & 5 & 5 & 4 \\
\hline$G$ & $C_{222}$ & $C_{321}$ & $C_{221}+\mathbf{1}$ & $C_{3}+C_{3}$ & $C_{4}+\mathbf{1}$ & $C_{5}$ & $C_{3}+2 \times \mathbf{1}$ & $4 \times \mathbf{1}$ \\
\hline
\end{tabular}

\section{Unimodular Subsystems of $D_{4}$}

The four-dimensional Voronoi polytope $P_{V}\left(D_{4}\right)$ is the self-dual regular fourdimensional polytope called the 24-cell. Coxeter [4] denotes it as $\{3,4,3\}$. According to (2), in the coordinates of Section 3, the edges of $P_{V}\left(D_{4}\right)$ are parallel either to the 4 vectors $e_{i}, 1 \leq i \leq 4$, or to the 8 vectors $\frac{1}{2}\left(e_{1} \pm e_{2} \pm e_{3} \pm e_{4}\right)$ for all 8 possibilities of signs. These 12 vectors up to the multiplier $\sqrt{2}$ are the 12 roots of a root system isomorphic to $D_{4}$. For convenience, we use below the usual form of the root system $D_{4}=\left\{e_{i} \pm e_{j}: 1 \leq i<j \leq 4\right\}$. Besides, we denote the vector $e_{i} \pm e_{j}$ by the symbol $i j^{ \pm}$, where the signs agree.

Note that $D_{4}$ consists of the following three 4-sets of mutually orthogonal vectors: $\left\{i j^{ \pm}, k l^{ \pm}\right\}$, where $\{i, j, k, l\}=\{1,2,3,4\}$. Call such a 4 -set of mutually orthogonal roots by a quadruple. Each quadruple relates to one of the three partitions of the 4-set $\{1,2,3,4\}$ into pairs. Call a three mutually orthogonal roots of $D_{4}$ by a triplet. Each triplet $t$ is contained in a quadruple $q_{t} \supset t$ which is uniquely 
determined by $t$. In other words, the triplet $t$ can be completed by a unique root $r_{t} \in D_{4}$ up to the quadruple $q_{t}$.

Consider the following two maximal unimodular systems contained in the root system $D_{4}$ : the graphic system $A_{4}-e$, where $e$ is any vector of $A_{4}$, and the cographic system $K_{3,3}^{*}$. The system $A_{4}-e$ represents the graphic matroid of the graph $K_{5}-1$, and the system $K_{3,3}^{*}$ represents the cographic matroid of the complete bipartite graph $K_{3,3}$.

It is not difficult to verify that each of these two unimodular subsystem of $D_{4}$ is a set $U=t_{1} \cup t_{2} \cup t_{3}$ of three mutually disjoint triplets. Label this set $U$ by the triad $\left(r_{1}, r_{2}, r_{3}\right)$ of the roots completing triples $t_{i}$ to the quadruples $q_{t_{i}}$. For example, the following set $U=\left\{i j^{-}, i j^{+}, k l^{-}: i j=12,13,14\right\}$ is labeled by the triad $\left(34^{+}, 24^{+}, 23^{+}\right)$.

The automorphism group of the root system $D_{4}$ consists of the following operations: permutations of indices, changing a pair of indices $(i j)$ by the complementing pair $(k l)$, reversing the sign of a unit vector $e_{i} \rightarrow-e_{i}$. The collection of all triads is partitioned into two orbits of the automorphism group. One orbit consists of triads with even number of minus signs. Another orbit contains triads with odd number of minus signs. The sets $U$ labeled by triads of these two orbits are isomorphic to unimodular systems $A_{4}-e$ and $K_{3,3}^{*}$, respectively.

There are many ways to choose in $D_{4}$ vectors forming the unimodular systems $A_{4}-e$ and $K_{3,3}^{*}$. We choose these vectors as follows. The 6 vectors $i j^{-}, 1 \leq$ $i<j \leq 4$, form the graphic system $A_{3}$ representing the graphic matroid of the complete graph $K_{4}$. If the vertices of $K_{4}$ are denoted by the numbers $1,2,3,4$, then the vector $i j^{-}=e_{i}-e_{j}$ represents the edge $(i j)$ connecting the vertices $i$ and $j$. Now suppose that the vertex 5 of $K_{5}-1$ is not connected with the vertex 1 of its subgraph $K_{4}$. Then we can relate the vectors $12^{+}, 13^{+}, 14^{+}$to the edges (25), (35), (45), respectively. It is easy to verify that the 9 vectors $i j^{-}$, $1 \leq i<j \leq 4,1 i^{+}, 2 \leq i \leq 4$, form the unimodular system $A_{4}-e$. This unimodular system consists of the following three triplets of mutually orthogonal vectors: $\left(i j^{-}, i j^{+}, k l^{-}\right), i j=12,13,14$.

The graph $K_{5}-1$ is planar. It has three vertices of degree 4 and two vertices of degree 3. The 9 edges of this graph are partitioned into the following two orbits (a) and (b) of the automorphism group of $K_{5}-1$ :

(a) 3 edges with both end vertices of degree 4; they are represented by the roots $23^{-}, 24^{-}, 34^{-}$

(b) 6 edges with end vertices of degree 3 and 4; they are represented by the roots $12^{ \pm}, 13^{ \pm}, 14^{ \pm}$

If we delete in the graph $K_{5}-1$ the edge (24), we obtain the planar graph $G_{5}:=K_{5}-2 \times 1$. The vertices $1,2,4,5$ of the graph $G_{5}$ have degree 3 and form a 4-cycle with edges (12), (25), (45), (14). The vertex 3 has degree 4 and 
it is adjacent to the four vertices of the 4-cycle by edges (13), (23), (35), (34). Now, we consider the dual graph $G_{5}^{+}$which is isomorphic to original one. Let the vertices of the 4-cycle of the $G_{5}^{+}$be $a, b, c, d$ in this order along the 4-cycle. Then edges of this 4-cycle, corresponding to the edges (13), (23), (35), (34) of $G_{5}$, have the following pairs of end vertices, respectively: $(a b),(b c),(c d),(a d)$. The fifth vertex $v$ of degree 4 of the graph $G^{+}$is adjacent to the vertices $a, b, c, d$ by edges, corresponding to the edges (14), (12), (25), (45) of $G_{5}$, respectively. The four last edges form a 4-cocycle of $G_{5}^{+}$. The 8 vectors $12^{-}, 13^{-}, 14^{-}, 23^{-}, 34^{-}, 12^{+}, 13^{+}$, $14^{+}$related to the edges of $G_{5}$ and $G_{5}^{+}$represent the graphic matroid of $G_{5}$ and the cographic matroid of $G_{5}^{+}$. The cographic matroid of $G_{5}^{+}$is a submatroid of $K_{3,3}^{*}$.

The graph $G_{5}^{+}$is obtained from $K_{3,3}$ by the contraction of one of its edges. The operation opposite to the contraction of an edge of the graph $K_{3,3}$ is the splitting of the vertex $v$ of degree 4 in $G_{5}^{+}$into two adjacent vertices $v^{\prime}$ and $v^{\prime \prime}$. This splitting is such that the vertex $v^{\prime}$ is adjacent to the vertices $a$ and $c$, and the vertex $v^{\prime \prime}$ is adjacent to the vertices $b$ and $d$. We obtain the complete bipartite graph $K_{3,3}$ with mutually non-adjacent vertices $b, d, v^{\prime}$ of one part and mutually non-adjacent vertices $a, c, v^{\prime \prime}$ of other part. The vectors related to edges $\left(a v^{\prime}\right),\left(c v^{\prime}\right)$ and $\left(b v^{\prime \prime}\right),\left(d v^{\prime \prime}\right)$ are $14^{-}, 12^{+}$and $12^{-}, 14^{+}$, respectively. Since $12^{+}-14^{-}=14^{+}-12^{-}=24^{+}$, we should relate the vector $24^{+}$to the edge $\left(v^{\prime} v^{\prime \prime}\right)$.

We see that this representation of the cographic matroid $K_{3,3}^{*}$ is obtained from the representation of the graphic matroid of the graph $K_{5}-1$ by changing the vector $24^{-}$into the vector $24^{+}$.

According to Theorem 1, the sum $P_{V}\left(D_{4}\right)+Z(Q)$ is a parallelotope only if the set $Q$ spans a unimodular system. According to Proposition 2, $Q \subseteq D_{4}$, since edges of $P_{V}\left(D_{4}\right)$ are parallel to roots. Hence, we have to find sbsets $Q \subseteq D_{4}$ spanning unimodular systems. It is sufficient to find maximal such sets.

Call a subset $Q \subseteq D_{4}$ spanning a unimodular system unextendable if the set $Q \cup\{r\}$ does not span a unimodular system for all $r \in D_{4}-Q$.

Proposition 3. Each unextendable subset of the root system $D_{4}$ is isomorphic to one of the following 3 subsets: $A_{4}-e, K_{3,3}^{*}$ and $q \cup\{r\}$, where $q$ is a quadruple and $r \in D_{4}-q$.

Proof. Let $q=\left\{i j^{-}, i j^{+}, k l^{-}, k l^{+}\right\} \subset D_{4}$ be a quadruple. Obviously it is a unimodular system. It is a basis of the space $\mathbf{R}^{4}$. For any root $r \in D_{4}-q$, the vector $2 r$ has \pm 1 coordinates in this basis. So, the system $q \cup\{2 r\}$ is unimodular for all $r \in D_{4}-q$. But it easy to verify that the set $q \cup\left\{2 r, 2 r^{\prime}\right\}$ does not spans a unimodular system for any $r^{\prime} \in D_{4}-(q \cup\{r\})$. Hence, each subset of $D_{4}$ of the form $q \cup\{r\}, r \notin q$, is an unextendable subset of $D_{4}$.

This implies that any other unextendable subset of $D_{4}$ does not contain a quadruple as a proper subset. Consider a set $U=t_{1} \cup t_{2} \cup t_{3}$ of three mutually disjoint 
triplets. Obviously, $U$ is a maximal subset of $D_{4}$ not containing a quadruple. (Note, there are $4^{3}=64$ such sets.) We saw that $U$ itself is a unimodular system isomorphic either to $A_{4}-e$, or to $K_{3,3}^{*}$. Obviously, these systems are unextendable.

Denote the zonotope related to a unimodular system $U$ by $Z(U)$. Since the deletion of the vector $24^{-}$from $A_{4}-e$ and the vector $24^{+}$from $K_{3,3}^{*}$ provides the same unimodular system $U\left(K_{5}-2 \times \mathbf{1}\right)$, the contraction of the edge zones of $Z\left(A_{4}-e\right)$ and $Z\left(K_{3,3}^{*}\right)$ corresponding to $24^{-}$and $24^{+}$, respectively, provides zonotopes, which are both isomorphic to $Z\left(U\left(K_{5}-2 \times \mathbf{1}\right)\right)$.

\section{Sums of $P_{V}\left(D_{4}\right)$ with Zonotopes}

The sums $P_{V}\left(D_{4}\right)+Z\left(A_{4}-e\right)$ and $P_{V}\left(D_{4}\right)+Z\left(K_{3,3}^{*}\right)$ are primitive parallelotopes. Their projections along a closed edge zone into three-dimensional space are drawn in Figs. II and III of [6]; see also [7]. Their Delaunay numbers are $N_{D}=2$ and $N_{D}=3$. In Figs. II and III, the closed edge zones of these parallelotopes are denoted by numbers $1,2, \ldots, 9$. The number 9 corresponds to the edge zone, along which the parallelotope is projected. Any non-primitive parallelotope is obtained from these two ones by contracting some closed edge zones. Some edges are denoted by $0 i, 1 \leq i \leq 8$. This means that after the contraction of the edge zone with number $i$ the edge with number $0 i$ is contracted to an edge of $P_{V}\left(D_{4}\right)$ which is denoted by 0 .

Figs. II and III show that the contraction of the edge zone 4 in $P_{V}\left(D_{4}\right)+$ $Z\left(A_{4}-e\right)$ and the edge zone 6 in $P_{V}\left(D_{4}\right)+Z\left(K_{3,3}^{*}\right)$ gives the same parallelotope. Comparing the numbers of edge zones of this parallelotope in Figs.II and III, we obtain their correspondence, shown in table below. Besides, this table gives the roots of the unimodular systems representing $A_{4}-e$ and $K_{3,3}^{*}$.

\begin{tabular}{|c||c|c|c|c|c|c|c|c|c|c|}
\hline Fig. I & 1 & 2 & 3 & 4 & 5 & 6 & 7 & 8 & 9 & - \\
\hline Fig. II & 2 & 1 & 5 & - & 7 & 8 & 3 & 4 & 9 & 6 \\
\hline roots & $23^{-}$ & $14^{-}$ & $12^{-}$ & $24^{-}$ & $34^{-}$ & $13^{-}$ & $12^{+}$ & $13^{+}$ & $14^{+}$ & $24^{+}$ \\
\hline
\end{tabular}

We see that the edge zones 1,4 and 5 of Fig.I contains edges of the same orbit (a) of the automorphism group of $K_{5}-\mathbf{1}$. Hence the contraction in $P_{V}\left(D_{4}\right)+Z\left(A_{4}-e\right)$ of any edge zone of this orbit gives isomorphic parallelotopes. The parallelotope with contracted edge zone 1 is the parallelotope of [6] with the Delaunay number $N_{D}=21$. This parallelotope is $P_{V}\left(D_{4}\right)+Z\left(U\left(K_{5}-2 \times \mathbf{1}\right)\right)$.

Note that for the zonotope $Z(U)$, which is a parallelotope, it is not important whether the summing vectors are orthogonal or not. A parallelepiped and a cube have the same L-type. But the orthogonality of summing vectors in $Z(U)$ affects 
heavily onto the L-type of the sum $P_{V}\left(D_{4}\right)+Z(U)$. This implied by the fact that any two orthogonal roots do not belong to a 2-face of $R_{V}\left(D_{4}\right)$. Hence the sum $P_{V}\left(D_{4}\right)+S(r)+S\left(r^{\prime}\right)$, where $r$ and $r^{\prime}$ are orthogonal, obtains a new 2-face spanned by $r$ and $r^{\prime}$ contrary to the case, when $r$ and $r^{\prime}$ are not orthogonal. (Here and below $S(r)$ is a segment of the line parallel to the root $r$.)

Facets of $P_{V}\left(D_{4}\right)$ are octahedra whose edges are parallel to roots of $D_{4}$. From now on, we sometimes identify edges and roots. Each octahedron has four pairs of opposite parallel triangle faces and six pairs of parallel edges, which represent six distinct roots. These six roots are partitioned into three pairs of orthogonal roots. Each triangle contains one representative root from these three pairs. Each of the six roots belongs to four triangles.

For a facet $F$ of $P_{V}\left(D_{4}\right)$, let $R(F)$ be the set of the six roots, which are edges of $F$. In this case, when edges are roots $e_{i} \pm e_{j}$, the facet vectors are parallel to the roots $\sqrt{2} e_{k}, 1 \leq k \leq 4$, and $\frac{1}{\sqrt{2}}\left(e_{1} \pm e_{2} \pm e_{3} \pm e_{4}\right)$. The facet vector $e_{k}, 1 \leq k \leq 4$, defines a facet $F$, such that $R(F)=\left\{i j^{ \pm}: i, j \neq k\right\}$. This is the root system $D_{3}$, which is isomorphic to $A_{3}$. The facet vector of another type $\frac{1}{2} \sum_{i=1}^{4} \varepsilon_{i} e_{i}$, where $\varepsilon_{i} \in\{ \pm 1\}$, defines a facet $F$, such that $R(F)=\left\{i j^{-\varepsilon_{i} \varepsilon_{j}}: 1 \leq i<j \leq 4\right\}$.

Note that, for each pair $\left(r, r^{\prime}\right)$ of orthogonal roots, there is a facet $F$ of $P_{V}\left(D_{4}\right)$, such that $r, r^{\prime} \in R(F)$. Recall that $P_{V}\left(D_{4}\right)$ has 24 facets (it is a 24-cell) and all its 16 belts are 3-belts.

Recall that $Z(U)=\sum_{r \in U} S(r)$ for any set of roots $U$.

Proposition 4. The following assertions hold.

(i) $P_{V}\left(D_{4}\right)+S(r)$ has no additional facets and belts for any root $r \in D_{4}$.

(ii) Let $P\left(r, r^{\prime}\right)=P V\left(D_{4}\right)+S(r)+S\left(r^{\prime}\right)$. If the roots $r, r^{\prime}$ are not orthogonal, then $P\left(r, r^{\prime}\right)$ has no additional facets and belts.

If $r$ and $r^{\prime}$ are orthogonal, then $P\left(r, r^{\prime}\right)$ has no additional facets, but it has one additional 2-belt.

(iii) For a triplet $t$ (of mutually ortogonal roots), two opposite shifts of the zonotope $Z(t)$ give two parallel facets of $P_{V}\left(D_{4}\right)+Z(t)$, whose facet vectors are the roots $\pm r_{t}$. These facets belong to 3 additional 3-belts of $P_{V}\left(D_{4}\right)+Z(t)$.

(iv) For any quadruple q, the polytope $P_{V}\left(D_{4}\right)+Z(q)$ is not a parallelotope.

\section{Proof.}

(i) Let $F$ be a facet of $P_{V}\left(D_{4}\right)$ and $r \notin R(F)$. Then $r$ does not lie in the hyperplane spanned by $F$. Hence, the facet $F$ remains a facet of $P_{V}\left(D_{4}\right)+$ $S(r)$. If $r \in R(F)$, then the facet $F$ is transformed to the facet $F+S(r)$ in the sum $P_{V}\left(D_{4}\right)+S(r)$. The facet $F+S(r)$ has also 4 pairs of parallel faces. But the four triangles of $F$ containing an edge parallel to $r$ are transformed into trapezoids with two edges parallel to $r$. 
(ii) Recall that there is a facet $F$ such that $r, r^{\prime} \in R(F)$. It is sufficient to consider such a facet.

If $r$ and $r^{\prime}$ are not orthogonal, then the facet $F+S(r)+S\left(r^{\prime}\right)$ has also 4 pairs of parallel faces. The two parallel faces which contain edges parallel to both the roots $r$ and $r^{\prime}$ are transformed into pentagons. The two pairs of faces having edges parallel only one of these two roots are transformed into two pairs of trapezoids. One pair of parallel faces is not changed.

If $r$ and $r^{\prime}$ are orthogonal, then each face of $F$ has only one edge parallel to one of these roots. Hence, each face is transformed into a trapezoid. There are two opposite vertices of $F$ which are not incident to the edges parallel to these roots. These vertices are transformed into new square faces $Q$ of $F+S(r)+S\left(r^{\prime}\right)$. So, this facet has now 10 faces. There are two pairs of opposite facets which are transformed into polyhedra with 10 faces. These four facets form a new 2-belt. So, each pair of orthogonal roots in $U$ generates a 2-belt.

(iii) Let $t=\left(r, r^{\prime}, r^{\prime \prime}\right)$. Consider the sum $P_{t}:=P\left(r, r^{\prime}\right)+S\left(r^{\prime \prime}\right)$. Let $F$ be a facet such that $r, r^{\prime} \in R(F)$. Then $r^{\prime \prime} \notin R(F)$. Let $Q$ be the quadrangle face of $F+S(r)+S\left(r^{\prime}\right)$. Then $Q+S\left(r^{\prime \prime}\right)$ is a cube. It is a new facet of $P_{t}$. This facet, its opposite and the facets of the 2-belt of $P\left(r, r^{\prime}\right)$ form a new 3-belt $\mathcal{B}$. But the cube $Q+S\left(r^{\prime \prime}\right)$ has 3 pairs of opposite faces, and each pair of its faces generates a 3-belt. Hence, for each $t$, the parallelotope $P_{t}$ has additionally three 3-belts.

The root $r_{t}$ is orthogonal to the new cubic facet $Q+S\left(r^{\prime \prime}\right)$. So, $r_{t}$ is the facet vector of this facet.

(iv) Obviously, $r_{t}$ is not orthogonal to any facet vector of the new 3-belt $\mathcal{B}$ of $P_{t}$. By Theorem 2, $P_{t}+S\left(r_{t}\right)$ is not a parallelotope.

Let $U=U(G)$ be a unimodular system of roots representing a subgraph $G \subseteq$ $K_{5}-1$ or $U=K_{3,3}^{*}$. Let $\pi(U)$ be the set of maximal pairs of orthogonal roots in $U$. A pair $\left(r, r^{\prime}\right) \subseteq U$ of orthogonal roots in $U$ is called maximal if there is no root in $U-\left\{r, r^{\prime}\right\}$ which is orthogonal to both the roots $r, r^{\prime}$. Let $\tau(U)$ be the set of triplets of mutually orthogonal roots in $U$. Recall that each triplet $t \in \tau(U)$ uniquely determines the fourth root $r_{t} \in D_{4}$, such that $r_{t}$ is orthogonal to all roots of $t$.

Proposition 5. Let $Z(U)=\sum_{r \in U} S(r)$. Then it holds:

(i) the sum $P_{V}\left(D_{4}\right)+Z(U)$ has $|\pi(U)|$ 2-belts and $16+3|\tau(U)|$ 3-belts;

(ii) the sum $P_{V}\left(D_{4}\right)+Z(U)$ has $24+2|\tau(U)|$ facets;

(iii) the sum $P_{V}\left(D_{4}\right)+Z(U)+S(r)$ is a parallelotope for all $r \in D_{4}$ such that $r \neq r_{t}, t \in \tau(U)$. 
Table 2. Zonotopes $Z(U)$ for $U \subseteq D_{4}$ such that $P_{V}\left(D_{4}\right)+Z(U)$ is a parallelotope

\begin{tabular}{|c|c|c|c|c|c|}
\hline$\overline{N_{D}}$ & $m$ & roots of the unimodular system $U$ & graph & $\operatorname{dim} U$ & $N_{D}^{0}$ \\
\hline- & 10 & $A_{4} \not \subset D_{4}$ cannot be added to $P_{V}\left(D_{4}\right)$ & $K_{5}$ & 4 & 1 \\
\hline 2 & 9 & $\left(12^{-}, 12^{+}, 34^{-}\right),\left(13^{-}, 13^{+}, 24^{-}\right),\left(14^{-}, 14^{+}, 23^{-}\right)$ & $K_{5}-\mathbf{1}$ & 4 & 4 \\
\hline 3 & 9 & $\left(12^{-}, 12^{+}, 34^{-}\right),\left(13^{-}, 13^{+}, 24^{+}\right),\left(14^{-}, 14^{+}, 23^{-}\right)$ & $K_{3,3}^{*}$ & 4 & 19 \\
\hline 20 & 8 & $\left(12^{-}, 12^{+}, 34^{-}\right),\left(13^{-}, 13^{+}, 24^{-}\right),\left(14^{+}, 23^{-}\right)$ & $K_{5}-\mathbf{2}$ & 4 & 6 \\
\hline 21 & 8 & $\left(12^{-}, 12^{+}, 34^{-}\right),\left(13^{-}, 13^{+}, 24^{-}\right),\left(14^{-}, 14^{+}\right)$ & $K_{5}-2 \times \mathbf{1}$ & 4 & 5 \\
\hline 22 & 7 & $\left(12^{+}, 34^{-}\right),\left(13^{-}, 13^{+}, 24^{-}\right),\left(14^{-}, 14^{+}\right)$ & $K_{5}-\mathbf{3}$ & 4 & 10 \\
\hline 23 & 7 & $34^{-},\left(13^{-}, 13^{+}, 24^{-}\right),\left(14^{-}, 14^{+}, 23^{-}\right)$ & $C_{2221}$ & 4 & 9 \\
\hline 24 & 7 & $\left(12^{-}, 12^{+}, 34^{-}\right),\left(13^{-}, 13^{+}, 24^{-}\right), 14^{+}$ & $K_{5}-\mathbf{1}-\mathbf{2}$ & 4 & 7 \\
\hline 25 & 7 & $\left(12^{+}, 34^{-}\right),\left(13^{-}, 13^{+}, 24^{-}\right),\left(14^{+}, 23^{-}\right)$ & $K_{4}+1$ & 4 & 8 \\
\hline 26 & 7 & $\left(12^{-}, 12^{+}, 34^{-}\right),\left(13^{-}, 13^{+}\right),\left(14^{-}, 14^{+}\right)$ & $K_{5}-\mathbf{1}-\mathbf{2}$ & 4 & 7 \\
\hline 27 & 6 & $\left(12^{-}, 12^{+}, 34^{-}\right),\left(13^{-}, 13^{+}\right), 14^{+}$ & $C_{321}$ & 4 & 12 \\
\hline 28 & 6 & $\left(12^{+}, 34^{-}\right),\left(13^{-}, 13^{+}, 24^{-}\right), 14^{+}$ & $C_{221}+\mathbf{1}$ & 4 & 13 \\
\hline 29 & 6 & $\left(13^{-}, 13^{+}, 24^{-}\right),\left(14^{-}, 14^{+}, 23^{-}\right)$ & $C_{222}$ & 4 & 11 \\
\hline 30 & 6 & $\left(12^{+}, 34^{-}\right),\left(13^{+}, 24^{-}\right),\left(14^{-}, 14^{+}\right)$ & $C_{221}+\mathbf{1}$ & 4 & 13 \\
\hline 31 & 6 & $\left(12^{-}, 12^{+}\right),\left(13^{-}, 13^{+}\right),\left(14^{-}, 14^{+}\right)$ & $C_{222}$ & 4 & 11 \\
\hline 32 & 6 & $\left(12^{+}, 34^{-}\right),\left(13^{-}, 24^{-}\right),\left(14^{-}, 14^{+}\right)$ & $C_{3}+C_{3}$ & 4 & 16 \\
\hline 33 & 6 & $\left(12^{+}, 34^{-}\right),\left(13^{+}, 24^{-}\right),\left(14^{+}, 23^{-}\right)$ & $K_{4}$ & 3 & $a_{1}$ \\
\hline 34 & 5 & $12^{+},\left(13^{-}, 13^{+}, 24^{-}\right), 14^{+}$ & $C_{3}+2 \times \mathbf{1}$ & 4 & 17 \\
\hline 35 & 5 & $\left(12^{-}, 12^{+}, 34^{-}\right), 13^{-}, 14^{+}$ & $C_{5}$ & 4 & 15 \\
\hline 36 & 5 & $\left(13^{-}, 13^{+}, 24^{-}\right),\left(14^{-}, 14^{+}\right)$ & $C_{4}+1$ & 4 & 14 \\
\hline 37 & 5 & $\left(12^{-}, 12^{+}\right),\left(13^{-}, 13^{+}\right), 14^{+}$ & $C_{4}+1$ & 4 & 14 \\
\hline 38 & 5 & $\left(12^{+}, 34^{-}\right),\left(13^{-}, 13^{+}\right), 14^{+}$ & $C_{3}+2 \times \mathbf{1}$ & 4 & 17 \\
\hline 39 & 5 & $\left(12^{+}, 34^{-}\right),\left(13^{+}, 24^{-}\right), 14^{+}$ & $C_{221}$ & 3 & $a_{2}$ \\
\hline 40 & 4 & $\left(13^{-}, 13^{+}, 24^{-}\right), 14^{+}$ & $4 \times \mathbf{1}=H_{4}$ & 4 & 18 \\
\hline 41 & 4 & $12^{+},\left(13^{-}, 13^{+}\right), 14^{+}$ & $4 \times \mathbf{1}=H_{4}$ & 4 & 18 \\
\hline 42 & 4 & $\left(12^{+}, 34^{-}\right), 13^{+}, 14^{+}$ & $C_{3}+1$ & 3 & $a_{3}$ \\
\hline 43 & 4 & $\left(13^{+}, 24^{-}\right),\left(14-, 14^{+}\right)$ & $4 \times \mathbf{1}=H_{4}$ & 4 & 18 \\
\hline 44 & 4 & $\left(13^{-}, 13^{+}\right),\left(14^{-}, 14^{+}\right)$ & $C_{4}$ & 3 & $a_{4}$ \\
\hline 45 & 3 & $\left(14^{-}, 14^{+}, 23^{-}\right)$ & $3 \times \mathbf{1}=H_{3}$ & 3 & $a_{5}$ \\
\hline 46 & 3 & $\left(13^{-}, 13^{+}\right), 14^{-}$ & $3 \times \mathbf{1}=H_{3}$ & 3 & $a_{5}^{\prime}$ \\
\hline 47 & 3 & $12^{+}, 13^{+}, 14^{+}$ & $3 \times \mathbf{1}=H_{3}$ & 3 & $a_{5}^{\prime \prime}$ \\
\hline St & 3 & $34^{-}, 13^{+}, 14^{+}$ & $C_{3}$ & 2 & $\alpha$ \\
\hline 48 & 2 & $\left(14^{-}, 14^{+}\right)$ & $2 \times \mathbf{1}=H_{2}$ & 2 & $\beta_{1}$ \\
\hline 49 & 2 & $13^{+}, 14^{+}$ & $2 \times \mathbf{1}=H_{2}$ & 2 & $\beta_{2}$ \\
\hline 50 & 1 & $14^{+}$ & $\mathbf{1}=H_{1}$ & 1 & \\
\hline 51 & 0 & & $24-$ cell & & \\
\hline
\end{tabular}

Table 2 illustrates Propositions 4 and 5. It shows the 35 unimodular systems $U$ of roots sums of which with $P_{V}\left(D_{4}\right)$ are parallelotopes. For the sake of completeness, in the first row of Table 2, we give the maximal unimodular system $A_{4} \not \subset D_{4}$, which cannot be added to $P_{V}\left(D_{4}\right)$. Maximal pairs and triplets of mutually orthogonal roots are, distinguished by parentheses. $H_{k}$ denotes the skeleton of a $k$-dimensional parallelepiped. As in Table 1, $N_{D}$ denotes the Delaunay number of the parallelotope 
$P_{V}\left(D_{4}\right)+Z(U)$ and $m$ is the number of roots in $U$. The parallelotope, missed by Delaunay and found by Stogrin, is denoted by St. The fifth column gives dimension of the added zonotope $Z(U)$. Note that $\operatorname{dim} Z(U)$ is equal to the rank of the graph $G$, which is represented by the unimodular system $U$.

In order to compare Tables 2 and 1, we add the last column. In this column $N_{D}^{0}$ denotes the Delaunay number $N_{D}$ of the corresponding zonotopal parallelotope $Z(U)$ if $\operatorname{dim} U=4 ; N_{D}^{0}=a_{i}, 1 \leq i \leq 5$, denotes a 3-dimensional zonotopal parallelotope if $\operatorname{dim} U=3$; and $N_{D}^{0}=\alpha, \beta_{1}, \beta_{2}$ denotes a 2-dimensional zonotopal parallelotope if $\operatorname{dim} U=2$. Note that

$a_{1}$ denotes a permutohedron $=$ a truncated octahedron;

$a_{2}$ denotes an elongated dodecahedron;

$a_{3}$ denotes a prism with a hexagonal base;

$a_{4}$ denotes a rhombic dodecahedron;

$a_{5}$ denotes a parallelopiped with mutually orthogonal edges;

$a_{5}^{\prime}$ denotes a parallelopiped with a pair of parallel rectangle facets;

$a_{5}^{\prime \prime}$ denotes a parallelopiped without rectangle facets;

$\alpha$ denotes a centrally simmetric hexagon;

$\beta_{1}$ denotes a rectangle;

$\beta_{2}$ denotes a parallelogram without orthogonal edges.

\section{REFERENCES}

1. M. Aigner, Combinatorial Theory, Springer-Verlag, Berlin, New-York, 1979.

2. J. H. Conway, assisted by R. Y. C. Fung, The Sensual (Quadratic) form, Math. Assoc. Amer., The Carus Math. Monographs, 261997.

3. J. H. Conway and N. J. A.Sloane, The cell structures of certain lattices, in: Miscellanea Mathematica, Springer-Verlag, Berlin, New York, 1991, pp. 71-107.

4. H. S. M. Coxeter, Regular polytopes, London, 1948.

5. V. I. Danilov and V. P. Grishukhin, Maximal unimodular systems of vectors, Europ. J. Combinatorics, 20 (1999), 507-526.

6. B. N. Delaunay, Sur la partition régulière de l'espace á 4 dimensions, Izvestia $A N$ SSSR, ser. phys.-mat., 1 (1929) 79-110 and 2 (1929), 145-164.

7. B. N. Delone (= B.N.Delaunay)), Geometry of positive quadratic forms, Uspekhi Math. Nauk, 4 (1938), (in Russian).

8. P. Engel, Geometric Crystallography. An axiomatic introduction to cristallography, D.Reidel Publishing Company, Dordrecht/ Boston/ Lancaster/ Tokyo, 1986.

9. P. Engel, On the symmetry classification of the four-dimensional parallelohedra, Zeitschrift für Kristallographie, 200 (1992), 199-213. 
10. V. P. Grishukhin, Parallelotopes of non-zero width, Math. Sbornik, 195(5) (2004), 59-78, (in Russian; translated in: Sbornik: Mathematics 195(5) (2004), 668-686).

11. P. McMullen, Space tiling zonotopes, Mathematika, 22 (1975), 202-211.

12. S. S. Ryshkov, On the structure of a primitive parallelohedron and on the last problem of Voronoi, Uspekhi Math. Nauk, 53 (1998), 161-162, (in Russian).

13. M. I. Stogrin, Regular Dirichlet-Voronoi partitions for the second triclinic group, Proc. Stekl. Inst. Math., 123, 1973.

14. F. Vallentin, Sphere coverings, lattices and tilings (in low dimensions), Dissertation, Technische Universität München, 2003.

15. B. A. Venkov, On a class of Euclidean polytopes, Vestnik of Leningradsk. Univ., 2 (1954), 11-31 (in Russian).

16. B. A. Venkov, On projecting of parallelohedra, Mat. Zametki, 49 (1959), 207-224 (in Russian).

17. G. F. Voronoi, Nouvelles applications des paramètres continus à la théorie de formes quadratiques - Deuxième mémoire. 2. Domaines de formes quadratiques correspondant aux différents types de paralléloèdres primitifs, $J$. für die reine und angewandte Mathematik, 136 (1909), 67-181.

\author{
M. Deza \\ ENS, Paris, \\ France \\ E-mail: deza@ens.fr \\ V. P. Grishukhin \\ CEMI RAN, \\ Moscow, Russia \\ E-mail: grishuhn@cemi.rssi.ru
}

